
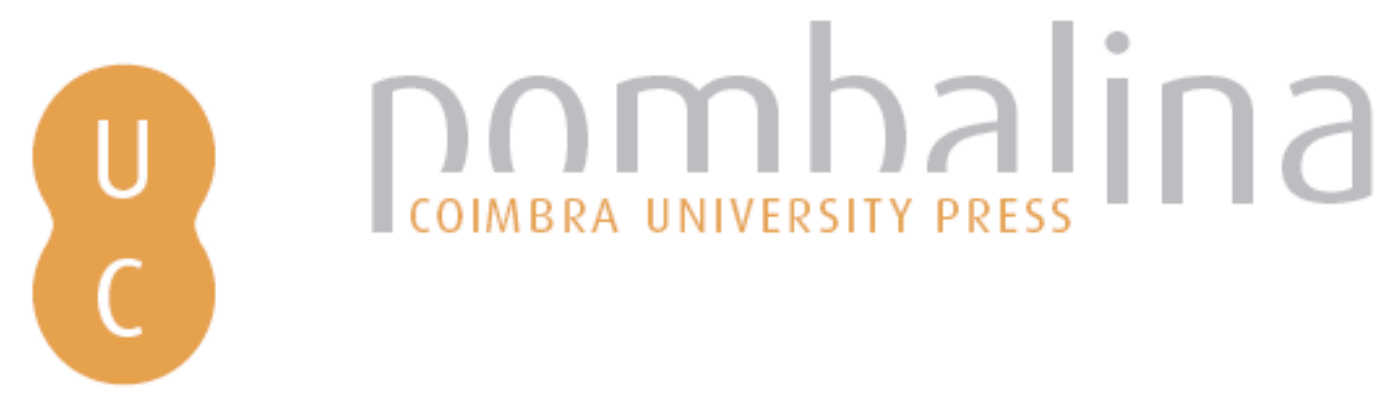

\title{
Integração de modelos de simulação em SIG: aplicação ao caso da drenagem de águas pluviais urbanas
}

\author{
Autor(es): $\quad$ Ribeiro, Alexandra; Cardoso, Alberto \\ Publicado por: Imprensa da Universidade de Coimbra \\ URL \\ persistente: URI:http://hdl.handle.net/10316.2/37091 \\ DOI: DOI:http://dx.doi.org/10.14195/978-989-26-0983-6_39 \\ Accessed : $\quad$ 26-Apr-2023 10:33:12
}

A navegação consulta e descarregamento dos títulos inseridos nas Bibliotecas Digitais UC Digitalis, UC Pombalina e UC Impactum, pressupõem a aceitação plena e sem reservas dos Termos e Condições de Uso destas Bibliotecas Digitais, disponíveis em https://digitalis.uc.pt/pt-pt/termos.

Conforme exposto nos referidos Termos e Condições de Uso, o descarregamento de títulos de acesso restrito requer uma licença válida de autorização devendo o utilizador aceder ao(s) documento(s) a partir de um endereço de IP da instituição detentora da supramencionada licença.

Ao utilizador é apenas permitido o descarregamento para uso pessoal, pelo que o emprego do(s) título(s) descarregado(s) para outro fim, designadamente comercial, carece de autorização do respetivo autor ou editor da obra.

Na medida em que todas as obras da UC Digitalis se encontram protegidas pelo Código do Direito de Autor e Direitos Conexos e demais legislação aplicável, toda a cópia, parcial ou total, deste documento, nos casos em que é legalmente admitida, deverá conter ou fazer-se acompanhar por este aviso.

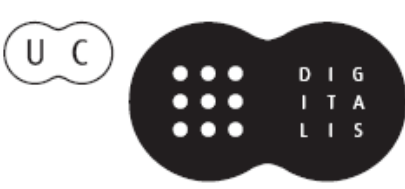




\section{$\forall$ \\ TAS DAS I JORNADAS LUSÓFONAS DE CIÊNCIAS E TECNOLOGIAS DE INFORMAÇÃO GEOGRÁFICA}

Editores

José Gomes dos Santos

Cidália Fonte

Rui Ferreira de Figueiredo

Alberto Cardoso

Gil Gonçalves

José Paulo Almeida

Sara Baptista 


\title{
ARTIGO 39
}

\section{INTEGRAÇÃO DE MODELOS DE SIMULAÇÃO EM SIG: APLICAÇÃo AO CASO da dRENAgEM de ÁguAs

\author{
PLUVIAIS URBANAS
}

RIBEIRO, Alexandra ${ }^{1,2}$ \& CARDOSO, Alberto $^{2}$

\begin{abstract}
1 Dep. Eng. Civil - Instituto Superior de Engenharia de Coimbra do Instituto Politécnico de Coimbra (Portugal); Instituto Superior de Engenharia de Coimbra; Rua Pedro Nunes - Quinta da Nora; 3030 -199 Coimbra, Portugal; Tel: +351 239790 200; Fax: +351 239790 221; email: alexr@isec.pt

2 Centro de Informática e Sistemas da Universidade de Coimbra (Portugal); Dep. Eng. Informática da Faculdade de Ciências e Tecnologia da Universidade de Coimbra Pólo II - Pinhal de Marrocos 3030 -290 Coimbra, Portugal; Tel: +351 239790 000; Fax: +351 239701 266; email: alberto@dei.uc.pt
\end{abstract}

\section{RESUMO}

Apesar da separação científica na maneira como os Sistemas de informação geográfica (SIG) e os modelos de simulação ambiental são construídos e utilizados, a necessidade contínua de estabelecer algum nível de integração entre ambos, para além da mera utilização de ambos, é conduzida pelo reconhecimento crescente que a avaliação integrada de todos os aspetos físicos, bióticos, sociais e económicos ambientais, é uma exigência do desenvolvimento sustentável. Pretende-se com este trabalho explorar as potencialidades de dois exemplos de software de SIG na integração dum modelo de simulação ambiental. Para tal, recorreu-se ao modelo Storm Water Management Model, um modelo de simulação hidrológica e hidráulica de sistemas de drenagem de águas pluviais urbanas, e ao software de SIG ArcGIS e QGIS. O primeiro é comercial e o segundo de código aberto. Apresenta-se aqui um exemplo de aplicação dum sistema de drenagem dual duma área residencial. O coletores foram dimensionados para um período de retorno de 2 anos, através de simulações iterativas. 
Atas das I Jornadas Lusófonas de Ciências e Tecnologias de Informação Geográfica, Sessão 9, Artigo 39 Integração de modelos de simulação em SIG: aplicação ao caso da drenagem de águas pluviais urbanas Alexandra Ribeiro \& Alberto Cardoso

\title{
PALAVRAS-CHAVE
}

Sistemas de informação geográfica, ArcGIS e QGIS, Integração, Modelação hidrológica e hidráulica, SWMM.

\section{INTEGRATION OF SIMULATION MODELS IN GIS: APPLICATION TO THE CASE OF URBAN STORM WATER DRAINAGE}

\begin{abstract}
Despite the scientific separation in how Geographic Information Systems (GIS) and environmental simulation models are constructed and used, the continuous need to establish some level of integration between the two, apart from the mere use of both, is driven by the growing recognition that the integrated assessment of all physical aspects, biotic, economic, social and environmental is a requirement of sustainable development. This work aims to explore the potential of two examples of GIS software in the integration of an environmental simulation model. For such, a model of hydrologic and hydraulic simulation of storm water drainage systems, the Storm Water Management Model, was used, and the GIS software ArcGIS and QGIS. The first is commercial and the second is open source. An example of application of dual drainage system in a residential area is presented here. The pipes were designed for a 2 -yr storm event through iterative simulations.
\end{abstract}

\section{KEYWORDS}

Geographic information systems, ArcGIS and QGIS, Integration, Hydrologic and hydraulic modelling, SWMM.

\section{INTRODUÇÃO}

Apesar da separação científica na maneira como os Sistemas de informação geográfica (SIG) e os modelos de simulação ambiental são construídos e utilizados, a necessidade contínua de estabelecer algum nível de integração entre ambos, para além da mera utilização de ambos, é conduzida pelo reconhecimento crescente que a avaliação integrada de todos os aspetos físicos, bióticos, sociais e económicos ambientais, é uma exigência do desenvolvimento sustentável (Clayton \& Radcliffe, 1996; Aspinall \& Pearson, 2000). 


\subsection{A integração de modelos de simulação ambientais e SIG}

A utilização conjunta dos SIG e dos modelos de simulação ambientais teve início por volta dos anos 80 do século XX (Brimicombe, 2010, cap. 7). As duas tecnologias são bastantes diferentes. Os SIG focam-se nas representações da localização, na distribuição espacial dos fenómenos/ entidades geográficas e nas relações entre estas no espaço. Essas são geralmente representações de carácter estático. Por sua vez, os modelos de simulação ambientais estão sobretudo interessados nos estados do sistema, em balanços de massa e na conservação da energia, ou seja, focados em quantidades (populações, produtos químicos e água) variáveis no tempo. No entanto, certamente com a ajuda dos SIG, e usando como exemplo o casos dos modelos hidrológicos, estes poderão mais facilmente passar do tratamento 1D das bacias de drenagem (e.g., cálculo de parâmetros espaciais médios), para uma abordagem de parâmetros espacialmente distribuídos. O cálculo de declives e da orientação de encostas, a delineação das bacias, a discretização do tipo e do uso do solo, o cálculo da interpolação de parâmetros com base em amostragens, e o delineamento de limites de cheia, tornam-se bastante facilitados pela utilização dos SIG.

Brandmeyer e Karimi (2000) propuseram a seguinte tipologia de integração: i) transferência de dados num sentido (one-way data transfer); ii) integração fraca (loose coupling); iii) integração partilhada (shared coupling); iv) integração por junção (joined coupling); e, integração de ferramentas (tool coupling). Na prática, o enfoque tem sido na integração fraca e na partilhada, onde as tecnologias (SIG e modelos de simulação) permanecem separadas. Em geral, apesar da existência da modelação ambiental dentro dos SIG, o papel predominante dos SIG na modelação ambiental tem sido para (Goodchild, 1993; Clark, 1998):

- Pré-processamento de dados geoespaciais com vista à sua entrada nos modelos de simulação. Isto envolve a integração de diferentes conjuntos de dados armazenados em formatos distintos, transformações, como sejam as de matricial para vetorial, operações de buffer, criação de novas layers por sobreposição, seleção e álgebra de mapas.

- Assistência nas tarefas de modelação, como sejam as tarefas de calibração e de construção de cenários. 
- Pós-processamento dos resultados da simulação para visualização e análise posterior usando processamento cartográfico ou álgebra de mapas, e.g., para determinar o impacto nos aglomerados urbanos, usos do solo, etc..

A integração dos modelos de simulação ambientais dentro dos SIG (integração por junção; joined coupling) tem beneficiado muito com os avanços nas linguagens de programação suportadas pelos SIG e com as ferramentas que estes disponibilizam para facilitar o uso dessas linguagens. Há uma forte tendência para a disponibilização de linguagens de scripting por parte dos SIG comerciais (e.g., ArcGIS, MapInfo, Geomedia) e não comerciais (e.g., QGIS, gvSIG, uDig), para além de outras linguagens de programação de alto nível (e.g., C\#.NET, VB.NET, Java). A utilização destas linguagens tornam a introdução dos dados, a visualização e a computação mais rápidas e fáceis do que a programação de raiz com base nas linguagens FORTRAN e C++. Outra direção pela qual algum software SIG tem enveredado, é a disponibilização de interfaces gráficas para a construção de modelos sob a forma de fluxogramas (cf. infra secção 3), o que permite ao utilizador encadear graficamente operações espaciais (e não só) em modelos de simulação.

\subsection{A integração de modelos de simulação hidrológica e hidráulica e} SIG: o caso particular da drenagem de águas pluviais urbanas

De acordo com Shamsi (2005, pág. 259-260), a integração dos modelos de simulação H\&H e os SIG segue uma tipologia com pontos comuns à apresentada na secção 1.1. Segundo este autor, a integração de modelos de simulação H\&H dentro do software SIG é o método que representa o relacionamento mais próximo entre as duas tecnologias, e fornece a base para um sistema de suporte à decisão para uma gestão efetiva dos sistemas de drenagem urbana.

Um software de modelação H\&H de sistemas de drenagem pluvial urbana de maior divulgação é o Storm Water Management Model (SWMM), da autoria da Environmental Protection Agency (EPA) dos EUA (cf. infra secção 2$)^{1}$. Trata-se dum software de acesso livre.

\footnotetext{
${ }^{1}$ Embora o SWMM tenha sido inicialmente desenvolvido para a modelação H\&H de sistemas de drenagem de águas pluviais urbanas, também é utilizado em situações de drenagem
} 
Existem várias soluções comerciais de integração do SWMM e de outro software do génerocom software SIG. Destacam-se as seguintes soluções: InfoSWMM, PCSWMM, MIKE Urban, SewerGEMS, XP-SWMM e SOBEK. Shamsi (2005, cap. 13) descreve vários outros exemplos de integração, nomeadamente entre o SWMM e o ArcView, precursor do software de SIG ArcGIS, do Environmental Systems Research Institute (ESRI).

Atualmente, alguns casos do software acima referido apresentam extensões para o ArcGIS.

Quanto à integração do SWMM em software open source, refere-se a iniciativa inpcom de integração dentro do gvSIG, e a iniciativa inp.PINS. Esta última consiste numa ferramenta cujo funcionamento se sintetiza da seguinte forma: i) gera o ficheiro de entrada para o SWMM a partir de dados SIG (shapefiles e um modelo digital do terreno em formato matricial); ii) manualmente, o utilizador abre a aplicação SWMM e preenche os restantes dados, aqueles que não estão associados a nenhuma entidade física ou geográfica, executando-a de seguida; iii) finalmente, combina os resultados do SWMM com o modelo digital do terreno, com vista à delineação dos limites de cheia no formato shapefile.

\subsection{Objetivo do trabalho}

Pretende-se com este trabalho explorar as potencialidades de dois exemplos de software de SIG na integração do SWMM (versão 5.0.022). Um dos casos é comercial e com forte penetração no mercado internacional, o ArcGIS (versão 10.2) da ESRI. O outro é de código livre, o QuantumGIS ou QGIS (versão 2.0.1 -Dufour), tendo, portanto, à partida a vantagem de ser gratuito.

Quer o ArcGIS quer o QGIS disponibilizam o Python como linguagem de scripting e um ambiente de geoprocessamento semelhante, o que facilita a integração do modelo H\&H dentro do SIG. Espera-se, ainda, que deste trabalho possam sair algumas pistas para futuras integrações de

de águas residuais domésticas e industriais, ou em situações em que ambas se combinam. 
modelos de simulação ambiental e SIG.

Este documento está estruturado da seguinte forma: na secção 2, apresentam-se as principais características do software SWMM; na secção 3, explica-se em termos gerais o ambiente de geoprocessamento do ArcGIS e do QGIS; na secção 4 resumem-se as fases de integração e compara-se a integração nos dois SIG; a secção 5 é dedicada ao exemplo de aplicação e à discussão dos resultados. Finalmente, na secção 6, apresentam-se as principais conclusões e o trabalho a desenvolver no futuro.

\section{O SOFTWARE SWMM}

O SWMM implementa um modelo dinâmico de precipitação -escoamento para a simulação da quantidade e da qualidade do escoamento superficial, especialmente em zonas urbanas. Tanto pode ser usado na simulação de redes de drenagem para um único evento pluvioso, como na simulação contínua ao longo do tempo (Rossman, 2009).

O modelo conceptual do SWMM está dividido em quatro compartimentos: atmosfera, superfície, subterrâneo e transporte. A precipitação é gerada no compartimento atmosfera e recebida pelo compartimento superfície; infiltra-se pelo compartimento subterrâneo ou é conduzida até ao compartimento transporte, o qual inclui coletores, canais, bombas, reguladores, unidades de armazenamento ou tratamento.

Este modelo conceptual é implementado através de um conjunto interligado de objetos, cada um com uma função específica. Os pluviómetros representam um dado evento pluvioso, descrito por ietogramas. As sub-bacias drenantes recebem esta precipitação e são caracterizadas por: área, largura, declive, percentagem de impermeabilidade, depressões de armazenamento e propriedades do solo. As sub-bacias podem ainda ser divididas em usos do solo específicos. Assim que a precipitação é recebida por uma sub-bacia, pode infiltrar-se de acordo com um destes métodos de infiltração: Horton, Green - Ampt ou SCS Curve Number. A água não infiltrada é transformada em escoamento superficial e é 
transportada através de uma rede de canais e coletores até uma saída final. Este transporte pode ser feito considerando os seguintes métodos de escoamento hidráulico: regime permanente, onda cinemática e onda dinâmica (ou difusa). A equação de Manning é utilizada para descrever a relação entre o caudal que passa nas condutas, a secção transversal, o raio hidráulico e o declive das mesmas. A poluição gerada pelo evento pluvioso é modelada recorrendo à concentração de poluentes e funções de propagação dos mesmos, definidas para cada tipo de uso do solo.

Algumas das entidades do modelo SWMM têm uma natureza física ou geográfica (e um conjunto inerente de atributos alfanuméricos), o que permite representá-las num mapa, e em particular num SIG.

A Figura 1 ilustra a forma como algumas dessas entidades físicas podem ser organizadas por forma a representar um sistema de drenagem.

O SWMM permite conhecer a evolução dos níveis de água e de qualidade da mesma em cada sub-bacia, assim como o caudal, a altura de escoamento e a qualidade da água em cada coletor e canal, durante um período de simulação composto por múltiplos intervalos de tempo (Rossman, 2009).

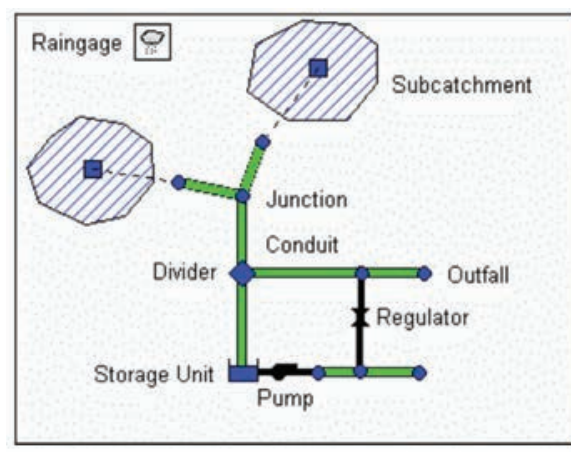

Figura 1 - Exemplo de entidades físicas do SWMM (figura retirada de Rossman, 2009) ${ }^{2}$

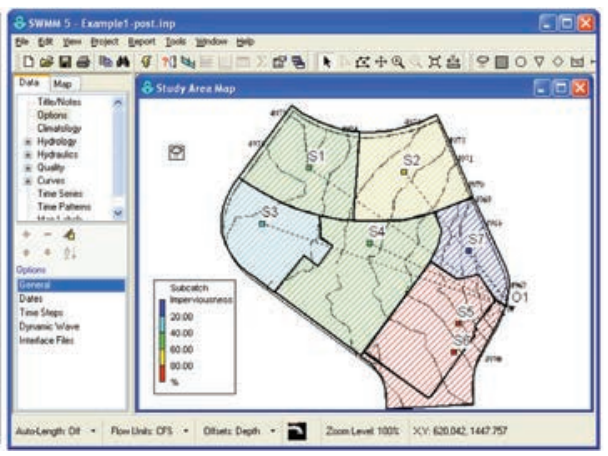

Figura 2 - Interface gráfica do SWMM 5

O SWMM foi desenvolvido para o sistema operativo Windows e apresenta uma interface gráfica (Figura 2) onde o utilizador pode: i) editar

\footnotetext{
2 raingage: pluviómetro; subcatchment: sub-bacia; junction: nó; storage unit: unidade de armazenamento; pump: bomba; regulator: regulador; conduit: coletor, canal natural ou artificial; outfall: saída do sistema
} 
manualmente os dados de entrada da área de estudo; ii) proceder a simulações hidrológicas, hidráulicas e de qualidade da água; iii) visualizar os resultados sob a forma de mapas, gráficos e tabelas; iv) e realizar análises estatísticas simples.

Para além da interface gráfica, é ainda disponibilizado um ficheiro executável (swmm5.exe) e um ficheiro dynamic link library (swmm5.dll). Estes podem ser integrados noutras aplicações e, desta forma, permitir a reutilização dos algoritmos do SWMM noutras plataformas de trabalho. Ambos recebem como entrada um ficheiro de dados em formato de texto e devolvem dois ficheiros, um de texto e outro binário. O ficheiro de texto contém o relatório da execução, incluindo mensagens de erro e tabelas de sumário dos resultados. O ficheiro binário contém os resultados duma simulação, em instantes predefinidos no ficheiro de entrada, para os elementos do sistema (coletores, canais, sub-bacias, bombas, etc.). É da responsabilidade da aplicação que interaja com o motor computacional do SWMM (.exe ou .dll) construir o ficheiro de entrada, caso não exista, aceder e mostrar os resultados contidos nos ficheiros de saída.

Juntamente com o ficheiro swmm5.dll, vem um conjunto de ficheiros com as declarações das funções presentes na biblioteca dll, para aplicações desenvolvidas em C/C++, Visual Basic for Applications (VBA) e Delphi. Existe um módulo Python (SWMM5_0.4.1.0; https://pypi.python.org/pypi/ SWMM5/), embora não da autoria da EPA, que possibilita o acesso às funções da biblioteca dll a partir de aplicações programadas em Python. Uma das vantagens da utilização desse módulo reside na facilidade de acesso ao ficheiro de resultados. O conteúdo deste ficheiro é rapidamente convertido em estruturas de dados do Python, nomeadamente listas, dicionários e geradores, que por sua vez poderão ser convertidas em dados reconhecidos pelo SIG (shapefiles, feature classes, tabelas alfanuméricas, ficheiros csv, etc.), através de scripts em Python.

\section{O AMBIENTE DE GEOPROCESSAMENTO DO ARCGIS E DO QGIS}

Existem bastantes semelhanças entre o ambiente de geoprocessamento 
do ArcGIS e o do QGIS. Os elementos fundamentais de ambos, muito idênticos, são: a ferramenta, a caixa de ferramentas, o modelo e construtor gráfico de modelos, o gestor do histórico de geoprocessamento, o processamento em batch e a linha de comandos de Python. Em ambos os casos, podem ser desenvolvidas ferramentas em Python (scripts). Os modelos não são mais que ferramentas encadeadas por forma a constituírem um fluxograma de trabalho. As ferramentas e os modelos podem ser parametrizados. Em função da tipologia dos parâmetros (e.g., ficheiro do tipo a ou b, layer, campo, valor escalar, etc.) assim o SIG gera a caixa de diálogo condizente.

Para além destes elementos, quer o ArcGIS quer o QGIS permitem a construção de add-ins (ou plug-ins), de maneira a incorporar funcionalidades ainda mais sofisticadas e facilitar a comunicação gráfica com o utilizador, e.g., um conjunto de ferramentas numa barra de ferramentas.

Uma característica fundamental do ambiente de geoprocessamento de ambos os SIG, é a possibilidade de construir novas ferramentas, modelos e plug-ins com base, não só, nas funcionalidades do próprio sistema, mas também noutras disponibilizadas por terceiros ou elaboradas pelo próprio utilizador para outras situações. A palavra chave é a reutilização.

\section{A INTEGRAÇÃo DO SWMM NO ARCGIS E NO QGIS}

Em ambos os casos, a integração do SWMM é feita recorrendo a ferramentas programadas em Python e a modelos. Não foram desenvolvidos plug-ins. Os modelos desenvolvidos incorporam as ferramentas desenvolvidas de raiz, ferramentas/add-ins do sistema e ferramentas/add-ins de terceiros.

A integração compreendeu as seguintes fases:

1. Conceção do modelo lógico geoespacial adequado ao SWMM. Consideraram-se as entidades físicas e não físicas e as relações entre si. Este modelo contempla não só as entidades que constituem a entrada no sistema, mas também as de saída.

2. Conversão do modelo lógico anterior para um modelo físico (ou 
de dados), baseado numa base de dados espacial relacional. No caso do ArcGIS utilizou-se a base de dados File Geodatabase (FGDB) e no caso do QGIS utilizou-se a base de dados PostgreSQL/PostGIS.

3. Importação de informação já existente para o modelo de dados anteriormente

definido. Nesta fase distinguiram-se duas situações:

3.1. Importação de dados provenientes do ficheiro de entrada (texto) do SWMM; desta forma é possível testar, no novo sistema, problemas anteriormente definidos no ambiente gráfico do SWMM.

3.2. Importação de informação relevante do cadastro da rede, por exemplo, em ficheiros CAD, shapefiles ou bases de dados.

4. Conversão do modelo de dados anterior para o ficheiro de entrada do SWMM.

5. Simulação com o motor computacional do SWMM e conversão dos resultados do SWMM (em ficheiro binário) para o modelo de dados definido na fase 1. Esta ferramenta utiliza o módulo Python SWMM5_0.4.1.0 referido na secção 2 .

6. Visualização e análise dos resultados recorrendo à combinação de diversas funcionalidades já existentes nos SIG, nomeadamente, simbologia, gráficos, perfis, animação gráfica e construção de relatórios, para visualização e comunicação do comportamento do sistema de drenagem ao longo do tempo.

A Figura 3 mostra o conjunto de ferramentas e modelos desenvolvidos para o ArcGIS e para o QGIS.

\subsection{Comparação da integração do SWMM no ArcGIS e no QGIS}

Procurou-se, sempre que possível, reutilizar os procedimentos relativos a cada fase nos dois SIG. No entanto, cada um dos SIG disponibiliza uma interface de programação de aplicativos (Application Programming Interface, API) diferente, embora na mesma linguagem Python, o que conduz a implementações distintas. Além disso, as bases de dados utilizadas num e noutro caso têm especificidades próprias que também se traduzem em diferenças na implementação. 


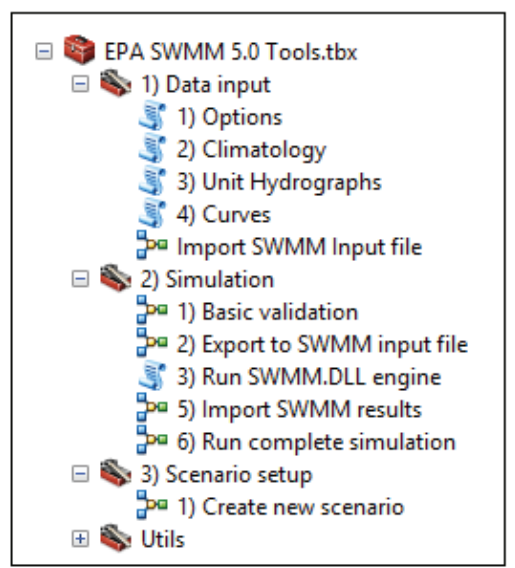

(a)

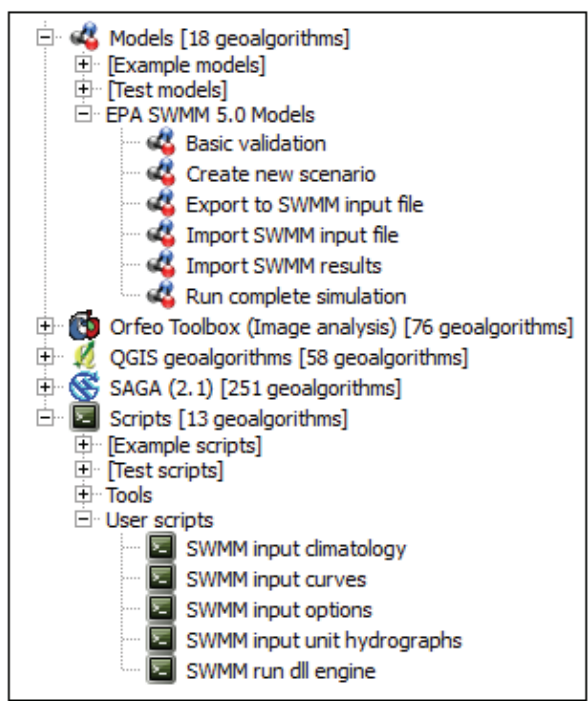

(b)

Figura 3 - Ferramentas e modelos desenvolvidos: (a) no ArcGIS; (b) no QGIS

A escolha de dois tipos diferentes de bases de dados e não apenas do PostGIS, deve-se ao facto de só recentemente a ESRI suportar este tipo. Verificou-se que a ligação e o acesso de leitura corriam bem, mas não se fizeram testes suficientes para garantir que todas as ferramentas do ArcGIS funcionariam de forma correta. Por outro lado, apesar de já existir uma File Geodatabase API livre, coloca-se o problema inverso no QGIS. O QGIS tem um bom suporte para o software livre, e, claro, a integração com o PostGIS é bastante boa. No entanto, apesar de ler a FGDB, detetaram-se alguns problemas ao nível da edição.

A forma de organizar e gerir as ferramentas no ArcGIS é mais intuitiva do que no QGIS (cf. supra Figura 3).

Embora a quantidade de ferramentas de edição geométrica do ArcGIS seja mais extensa, as do QGIS são bastante intuitivas e foram suficientes. Ambos os SIG possuem ferramentas de edição e validação topológica, necessárias para verificação da conectividade da rede antes de se gerar o ficheiro de entrada do SWMM. As do ArcGIS são mais completas, mas as do QGIS foram suficientes. Não foi utilizada nenhuma funcionalidade para a análise de redes. 
O suporte de edição das tabelas de atributos (e outras) é semelhante nos dois casos, embora o QGIS apresente a vantagem de permitir a edição de tabelas na forma de "formulário", o que em alguns casos é mais agradável para o utilizador. O preenchimento dos campos obrigatórios do modelo de dados desenvolvido com base em informação contida noutros campos, e a junção/relação de tabelas, são suportados em ambos os casos.

\section{EXEMPLO DE APLICAÇÃO}

\subsection{Descrição}

Os testes foram realizados com base em 9 exemplos (Gironás, 2009). O SWMM está preparado para modelizar um conjunto muito variado de situações e esses 9 exemplos refletem essa variedade. Para garantir que a integração é bem sucedida, isto é, que conduz aos mesmos resultados das simulações realizadas exclusivamente no SWMM, optou-se pela utilização destes exemplos. Cada exemplo vem acompanhado de um ficheiro de dados em formato texto. Neste trabalho apresenta-se apenas um desses exemplos de aplicação (Gironás, 2009, pág. 118 -127).

O exemplo considerado diz respeito a uma zona residencial com uma área aproximada de 29 acre $(\cong 11.7$ ha). A zona foi dividida em 7 sub-bacias irregulares para melhor capturar o efeito da variabilidade da topografia, do tipo de cobertura e de características do solo, na geração de escoamento superficial.

O objetivo deste exemplo é simular um sistema de drenagem dual (AMK Associates, 2004). Estes sistemas têm duas partes distintas: “(1) uma superficial, sistema major ou principal, composta por ruas, canais naturais e artificiais, depressões e zonas de acumulação de água, entre outros; (2) uma rede de coletores, designada por sistema minor ou secundário. Quando a capacidade de carga do sistema de coletores é ultrapassada, a água sai dos coletores através das sarjetas, sumidouros e câmaras de visita, surgindo à superfície. Este volume de água em excesso pode ficar acumulado em pontos baixos, ser infiltrado, entrar novamente no sistema de coletores ou originar escoamento superficial" (Marques et al., 
2013, pág. 33). Habitualmente, o sistema de coletores é projetado para um período de retorno de 2 a 10 anos, enquanto o sistema de drenagem superficial pode ser projetado para lidar com eventos de 25 a 100 anos (Smith, 2006).

Neste exemplo, o sistema secundário é representado pelos coletores e sarjetas das ruas. Se este sistema entrar em sobrecarga, as ruas serão inundadas. O sistema principal é representado pelo "escoamento nas ruas" e pelo canal que atravessa o parque da zona. A rede de coletores foi dimensionada para um evento pluvioso de 2 anos. Resumindo, estudaram-se dois cenários de simulação:

Tabela 1 - Cenários de simulação; os aspetos que distinguem os cenário 1 do cenário base estão assinalados a negrito.

\begin{tabular}{|c|c|c|c|}
\hline \multicolumn{2}{|c|}{ Cenário base } & \multicolumn{2}{c|}{ Cenário 1 } \\
\hline \multicolumn{2}{|c|}{ Período de retorno 2 anos } & \multicolumn{2}{c|}{ Período de retorno 2 anos } \\
\hline Coletor & Diâmetro (ft) & Coletor & Diâmetro (ft) \\
\hline P1 & 1 & P1 & 1.33 \\
\hline P2 & 1 & P2 & 1.5 \\
\hline P3 & 1 & P3 & 1.5 \\
\hline P4 & 3 & P4 & 1.67 \\
\hline P5 & 3 & P5 & 1.83 \\
\hline P6 & 3 & P6 & 2 \\
\hline P7 & 3 & P7 & 2 \\
\hline P8 & 3 & P8 & 3.17 \\
\hline
\end{tabular}

A Figura 4 mostra as opções de análise consideradas para ambos os cenários. A Figura 5 mostra o ietograma (precipitação vs tempo) respeitante ao evento de 2 anos. Na Figura 6 é representado o cenário base de simulação, nomeadamente, todas as layers correspondentes às entidades físicas deste sistema de drenagem e algumas das respetivas tabelas de atributos, quer no ArcGIS quer no QGIS. 
Flow Units ........... CFS

Process Models:

Rainfall/Runoff ... YES

snowmelt ............

Groundwater ........ NO

Flow Routing .......YES

Ponding Allowed ...NO

Water Quality .....NO

Infiltration Method ..HORTON

Flow Routing Method ...DYNWAVE

Starting Date ........JAN-01-2007

$$
00: 00: 00
$$

Ending Date .........JAN-01-2007

$12: 00: 00$

Antecedent Dry Days ..0.0

Report Time step ....00:01:00

Wet Time Step .......00:01:00

Dry Time step ......01:00:00

Routing Time step ...15.00 sec

Figura 4 - Opções de análise dos cenários

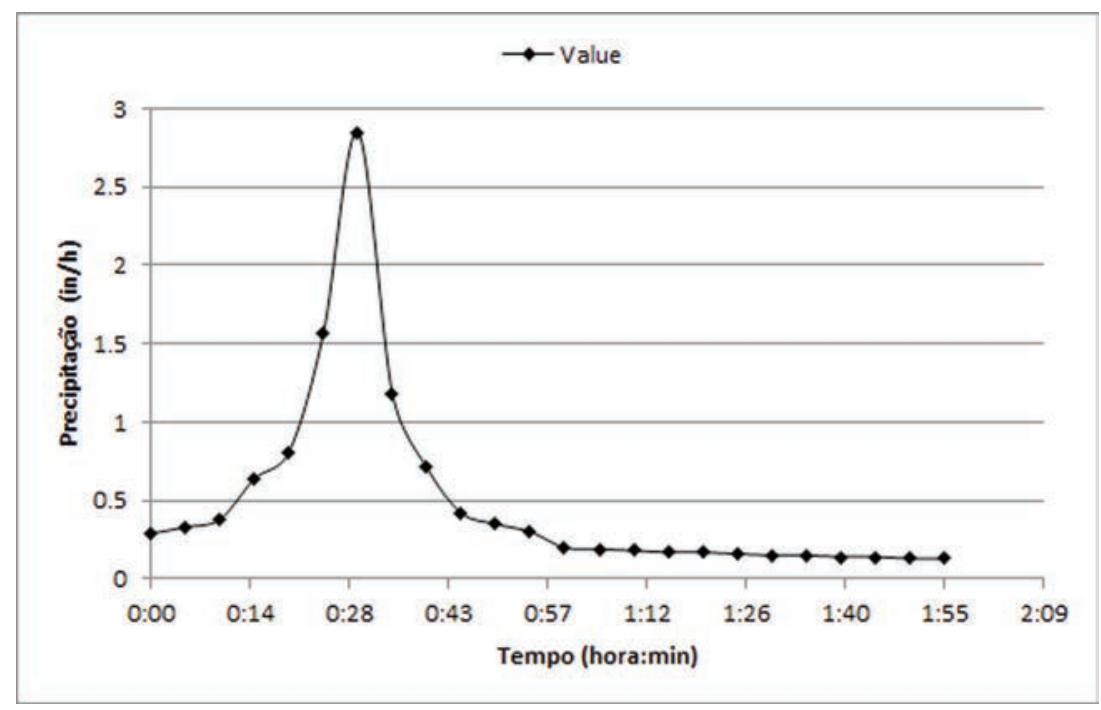

Figura 5 - Ietograma do evento com um período de retorno de 2 anos 


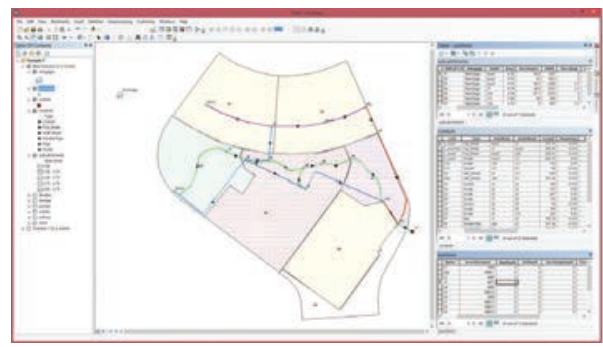

(a)

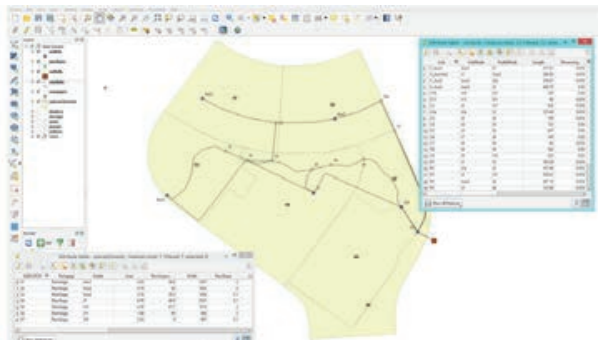

(b)

Figura 6 - Layers correspondentes ao cenário base e algumas das tabelas de atributos com dados de entrada: (a) no ArcGIS; (b) no QGIS

\subsection{Discussão dos resultados}

\subsubsection{Resultados da simulação}

\section{Cenário base:}

Os resultados da resolução do cenário base indicam que não há nós em sobrecarga ou inundados, pois as tabelas correspondentes às secções Node Surcharge Summary e Node Flooding Summary do relatório do SWMM vêm vazias. Contudo, os coletores P1, P2 e P3, entraram em sobrecarga, pois a tabela sumário de sobrecargas de condutas assim o indica (Tabela 2). Isto significa que o sistema de coletores se encontra subdimensionado.

Tabela 2 - Tabela sumário de sobrecarga de condutas

res_conduit_surcharge_sum
\begin{tabular}{|l|r|r|r|r|r|r|}
\hline Link & HoursFullBothEnds & HoursFullUpstream & HoursFullDnstream & Hours_AboveNormalFlow & HoursCapacityLimited \\
\hline P1 & 0.01 & 0.01 & 0.01 & 0.2 & 0.01 \\
\hline P2 & 0.3 & 0.3 & 0.31 & 0.31 & 0.24 \\
\hline P3 & 0.2 & 0.2 & 0.21 & 0.34 & 0.19 \\
\hline
\end{tabular}

\section{Cenário 1:}

O diâmetro das condutas foi iterativamente modificado por forma a obter uma solução onde os coletores não entrassem em sobrecarga, com um fator de segurança de, pelo menos, $15 \%$, i.e., um rácio altura do coletor/altura de água inferior a 85\% (Tabela 3, campo RatioMaxFullDepth). A mesma tabela (campo MaxVelocity) mostra que o critério da velocidade máxima nos coletores é verificado $(10 \mathrm{ft} / \mathrm{s})$. 
De acordo com os critérios de drenagem, num evento de 2 anos não deve haver inundação das sarjetas das ruas (C2a, C2, C_Aux1, C_Aux2, C_Aux1to2). Tendo em conta as características das secções transversais consideradas para as sarjetas, se o rácio anterior for inferior a 0.38 , não há risco da água galgar os passeios (Tabela 3). Logo, o sistema é apropriado em termos de fluxos de água nas ruas.

\begin{tabular}{|c|c|c|c|c|c|c|c|c|}
\hline \multicolumn{9}{|c|}{ res_link_flow_sum } \\
\hline & Link & Type & Maxflow & DaysMaxOc & HoursMaxOcc & MaxVelocity & RatioMaxfullflow & RatioMaxFullDepth \\
\hline 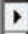 & $\mathrm{C} 2 \mathrm{a}$ & CHANNEL & 0 & 0 & 12:00:00 AM & 0 & 0 & 0 \\
\hline & C2 & CHANNEL & 0 & 0 & 12:00:00 AIM & 0 & 0 & 0 \\
\hline & C3 & CONDUIT & 0 & 0 & 12:00:00 AM & 0 & 0 & 0 \\
\hline & $\mathrm{C4}$ & CONDUIT & 0 & 0 & 12:00:00 AM & 0 & 0 & 0 \\
\hline & C5 & CONDUIT & 0 & 0 & 12:00:00 AM & 0 & 0 & 0 \\
\hline & C6 & CONDUT & 0 & 0 & 12:00:00 AM & 0 & 0 & 0 \\
\hline & C7 & CONDUT & 0 & 0 & 12:00:00 AM & 0 & 0 & 0 \\
\hline & C8 & CONDUIT & 0 & 0 & 12:00:00 AM & 0 & 0 & 0 \\
\hline & C9 & CONDUT & 0 & 0 & $12: 00: 00 \mathrm{AM}$ & 0 & 0 & 0 \\
\hline & C10 & CONDUT & 0 & 0 & 12:00:00 AM & 0 & 0 & 0 \\
\hline & C11 & CONDUT & 41.45 & 0 & 12:35:00 AM & 8.76 & 0.21 & 0.31 \\
\hline & C_Aux1 & CHANNEL & 6.59 & 0 & 12:35:00 AM & 2.17 & 0.03 & 0.27 \\
\hline & C_Aux2 & CHANNEL & 7.65 & 0 & 12:35:00 AM & 2.17 & 0.03 & 0.29 \\
\hline & C_Aux1to2 & CHANNEL & 0 & 0 & 12:00:00 AM & 0 & 0 & 0.15 \\
\hline & C_Aux3 & CONDUT & 0 & 0 & 12:00:00 AM & 0 & 0 & 0 \\
\hline & P1 & CONDUT & 6.58 & 0 & 12:35:00 AM & 6.24 & 0.81 & 0.71 \\
\hline & P2 & CONDUIT & 7.66 & 0 & 12:35:00 AM & 6.11 & 0.86 & 0.67 \\
\hline & P3 & CONDUT & 7.51 & 0 & 12:36:00 AM & 5.05 & 0.72 & 0.8 \\
\hline & P4 & CONDUT & 4.2 & 0 & 12:35:00 AM & 3.08 & 0.47 & 0.64 \\
\hline & PS & CONDUIT & 6.54 & 0 & 12:36:00 AM & 3.64 & 0.57 & 0.65 \\
\hline & P6 & CONDUIT & 9.79 & 0 & 12:36:00 AM & 4.21 & 0.83 & 0.72 \\
\hline & P7 & CONDUT & 18 & 0 & 12:35:00 AM & 6.64 & 0.92 & 0.81 \\
\hline & P8 & CONDUT & 29.33 & 0 & 12:35:00 AM & 7.32 & 0.63 & 0.51 \\
\hline
\end{tabular}

Tabela 3 - Tabela sumário do escoamento nos arcos (coletores, sarjetas, canais)

Para além das tabelas exibidas anteriormente, as bases de dados construídas suportam outras tabelas de resultados do SWMM, nomeadamente, tabelas com variáveis relativas a sub-bacias, nós e arcos (coletores, sarjetas, canais) para cada instante reportado pelo SWMM (Figura 7). A partir desta informação é possível mostrar visualmente, nos dois SIG, a evolução temporal destas variáveis. 


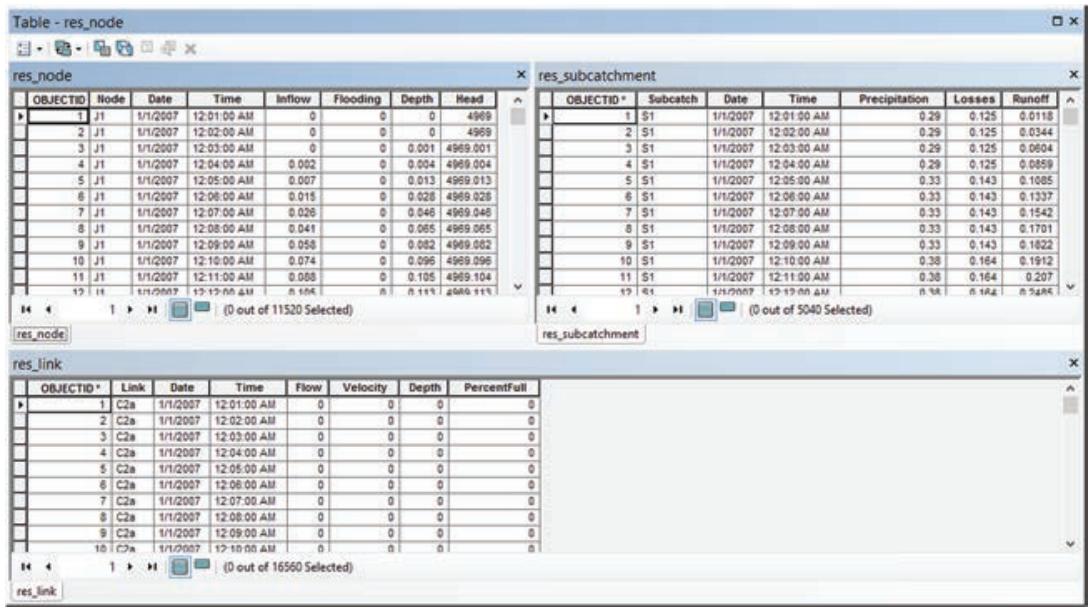

Figura 7 - Tabelas de resultados dos nós, arcos e sub-bacias para cada instante

\subsubsection{Resultados da integração}

Para além dos resultados da simulação, importa discutir os resultados da integração propriamente dita. O desenvolvimento das ferramentas no ArcGIS e no QGIS foi feito com base nos site packages ArcPy e PyQGIS, respetivamente. É através destes "pacotes de módulos" que se pôde aceder programaticamente às funcionalidades dos dois SIG, usando a linguagem Python. Quer num caso quer noutro, foram usadas classes e funções que permitiram executar tarefas essenciais como: i) aceder a layers no mapa; ii) carregar layers a partir de fontes de dados vetoriais e rasters em diferentes formatos; iii) fazer inquirições; iv) selecionar subconjuntos de entidades (features); v) aceder à geometria e aos atributos das entidades; vi) realizar cálculos com base em atributos ou na geometria; vii) adicionar, apagar e calcular campos; viii) realizar operações espaciais do tipo união, interseção, recorte, etc.; ix) e criar novas entidades. Além disso, pelo facto da linguagem de programação ser a mesma, foi possível a aceder aos mesmos módulos disponibilizados por terceiros (e.g., NumPy).

No entanto, notaram-se alguns pontos menos bons do QGIS relativamente ao ArcGIS:

- A documentação no ArcGIS está centralizada e é bastante detalhada, algo que não acontece com o QGIS. 
- A tipologia de parâmetros de entrada e de saída que se pode considerar em cada ferramenta é menor no caso do QGIS do que no caso do ArcGIS; isto significa que é necessário escrever mais código para realizar a validação dos parâmetros.

- Para algumas operações foi necessário escrever mais código no QGIS porque não existiam ainda ferramentas pré-programadas.

\section{CONCLUSÕES E TRABALHO FUTURO}

Neste trabalho integrou-se um modelo de simulação hidrológica e hidráulica de redes de drenagem de águas pluviais urbanas - o SWMM -, em dois exemplos de software SIG amplamente utilizados a nível internacional - o ArcGIS e o QGIS. Ambos os SIG possuem um ambiente de geoprocessamento e um conjunto de outras funcionalidades (simbologia, gráficos, perfis, animação gráfica) que facilitaram a integração pretendida. Embora hajam diferenças no processo de integração, o resultado final foi muito semelhante.

Há, no entanto, muitas melhorias a introduzir nos sistemas desenvolvidos. Destas, destacam-se as seguintes:

- Melhorar a comunicação com o utilizador, o que inclui a construção de mais ferramentas/modelos para automatizar algumas das tarefas atuais (e.g., junções e relações de tabelas, campos calculados, construção de modelos para gráficos). Um utilizador pouco familiarizado com os SIG poderá ter algumas dificuldades. Será, eventualmente, necessário criar alguns add-ins ou plug-ins a partir do trabalho desenvolvido.

- Melhorar a construção e a gestão de cenários de simulação no sentido de evitar a duplicação de informação.

Por outro lado, pretende-se ainda:

- Testar com um caso real, onde a delineação das sub-bacias se faça de forma automática e se utilizem as funcionalidades dos SIG para a extração de parâmetros relativos a todos os aspetos da modelação H\&H de sistemas de drenagem de águas pluviais urbanas. 
- Integrar os resultados do SWMM num modelo de delineação de zonas de cheia.

\section{BIBLIOGRAFIA}

AMK Associates, International, Ltd. (2004) - Dual drainage storm water management model, Program Documentation and Reference Manual, Release 2.1, Ontário, Canadá. ArcGIS. Redlands, CA: Environmental Systems Research Institute (ESRI). Retirado de http://www.esri.com [Acedido em 20/03/2014].

ASPINALL, Richard, \& PEARSON, Diane (2000) - "Integrated geographical assessment of environmental condition in water catchments: Linking landscape ecology, environmental modelling and GIS". Journal of Environmental Management 59: 299-319.

BRANDMEYER, Jo Ellen \& KARIMI, Hassan (2000) - "Coupling methodologies for environmental models”. Environmental Modelling \& Software 15: 479-488.

BRIMICOMBE, Allan (2010) - "GIS, environmental modeling and engineering" ( $2^{\mathrm{a}}$ ed.). Boca Raton: CRC Press/Taylor \& Francis Group.

BRIMICOMBE, Allan J \& LI, Yang \& AL -ZAKWANI, Abdullah \& LI, Chao (2009) - "Agent - based distributed component services in spatial modeling". In Computational science and its applications-ICCSA 2009, ed. O. Gervasi et al., 300-312. Berlin: Springer -Verlag.

CLARK, Michael (1998) - "Putting water in its place: A perspective on GIS in hydrology and water management”. Hydrological Processes, 12: 823-834.

CLAYTON, Anthony \& RADCLIFFE, Nicholas (1996) - Sustainability: A systems approach. London: Earthscan Publications.

FEDRA, Kurt (1993). "GIS and environmental modeling”. In Environmental modeling with GIS, ed. M.F. Goodchild, B.O. Parks, \& L.T. Steyaert, 35-50. New York: Oxford University Press.

GIRONÁS et al. (2009) - Storm water management model applications manual. National Risk Management Research Laboratory, Office of Research and Development, US Environmental Protection Agency.

GOODCHILD, Michael (1993) - "The state of GIS for environmental problem -solving”. In Environmental modeling with GIS, ed. M.F. Goodchild, B.O. Parks, \& L.T. Steyaert, 8-15. New York: Oxford University Press.

MARQUES, Alfeu, et al. (2013) - Hidrologia Urbana - Sistemas de Drenagem de águas pluviais urbanas. Entidade Reguladora dos Serviços de Águas e Resíduos. Universidade de Coimbra. 
MCDONNELL, Rachael (1996) - "Including the spatial dimension: Using geographical information systems in hydrology". Progress in Physical Geography 20: 159-177.

QGIS Development Team (2014) - QGIS Geographic Information System. Open Source Geospatial Foundation Project. Retirado de http://qgis.osgeo.org [Acedido em 20/03/2014].

ROSSMAN, Larry (2009) - Storm Water Management Model User's Manual Version 5.0. EPA/600/R -05/040, U.S. Environmental Protection Agency, National Risk Management Research Laboratory, Cincinnati, OH.

SENGUPTA, Raja, \& BENNETT, David (2007) - "Agent -based modelling environment for spatial decision support”. International Journal of Geographic Information Science 17: 157-180.

SHAMSI, Uzair (2005) - GIS applications for water, wastewater, and stormwater systems. CRC Press.

SMITH, Michael (2006) - "Comment on Analysis and modeling of flooding in urban drainage systems”, Journal of Hydrology, 317(3 -4), pp. 355 -363. Storm Water Management Model (SWMM). USA Environmental Protection Agency (EPA). Retirado de http://www.epa.gov/nrmrl/wswrd/wq/models/ swmm/ [Acedido em 20/03/2014].

TSOU, Ming -Hsiang, \& BUTTENFIELD, Barbara (2002) - "A dynamic architecture for distributed geographic information services". Transactions in GIS 6: 355-381. 
Série Documentos

Imprensa da Universidade de Coimbra

Coimbra University Press

2015

- U M

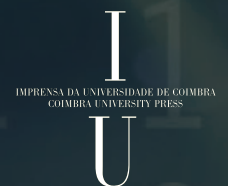

